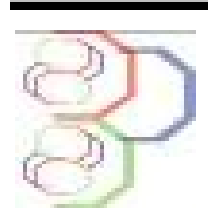

\title{
Caractérisation de quelques peuplements naturels de Baobab (Adansonia digitata L.) et des pressions subies dans les différentes zones chorologiques du Benin
}

\author{
Dossa K..$^{*}$, Toni H. ${ }^{1}$, Azonanhoun P. ${ }^{1}$, Djossa A.B. ${ }^{1,2}$ \\ 'Laboratoire d'Écologie Appliquée (LEA), Faculté des Sciences Agronomiques (FSA), Université d'Abomey-Calavi \\ (UAC), Bénin. 01 BP 526 Cotonou, Bénin. \\ 'Ecole Nationale Supérieure des Sciences et Techniques Agronomiques de Kétou, Université d'Agriculture de Kétou, \\ Bénin \\ ${ }^{*}$ Correspondance author : dossakomivi@gmail.com , Nom : DOSSA Komivi
}

Original submitted in on 28 ${ }^{\text {th }}$ March 2015. Published online at www.m.elewa.org on 30th September 2015 http://dx.doi.org/10.4314/iab.v93i1.9

\section{RÉSUMÉ}

Objectif: Le Baobab est une espèce utilisée à diverses fins par les populations locales au Bénin. Cependant, l'état des peuplements de l'espèce reste peu connu dans plusieurs localités du pays. Cette étude a permis de caractériser les peuplements de baobab dans les communes de Dassa, Matéri et Comè situées dans les trois zones chorologiques du Bénin

Méthodologie et Résultat : Six cent soixante six (666) pieds de baobab ont été recensés et étudiés dans les 3 communes afin de mesurer des paramètres dendrométriques comme le $\mathrm{DBH}$, la hauteur et le diamètre du houppier des arbres des peuplements et d'établir le type de distribution spatiale des individus au sein de chaque peuplement. Une enquête auprès de 300 personnes au sein des communautés riveraines des peuplements étudiés a permis de recenser les diverses pressions anthropiques auxquelles l'espèce est soumise. Les résultats ont révélé que les peuplements de baobab étudiés sont globalement dominés par des individus de grande taille (hauteur : $15-20 \mathrm{~m}$ et DBH : 100-150 cm avec de larges houppiers $(5-10 \mathrm{~m}$ ) et une faible représentation des individus de la régénération $(\mathrm{DBH} \leq 50 \mathrm{~cm})$. La structure spatiale épouse une distribution globalement aléatoire. La faible régénération est surtout rapportée dans les jeunes jachères et les champs. Les formes et l'intensité des pressions anthropiques qui pèsent sur l'espèce varient d'une commune à l'autre, mais l'agriculture extensive constitue la principale menace rapportée.

Conclusion et application : La survie à long terme des peuplements de baobab étudiés est compromise, si la tendance de la faible régénération observée se maintient. Des efforts doivent donc être fournis pour lever les diverses pressions qui pèsent sur l'espèce et surtout favoriser la régénération. Les résultats de ces travaux préliminaires, posent les bases pour des études ultérieures de suivi régulier de ces peuplements afin d'analyser leur évolution dans le contexte actuel des pressions anthropiques encourues et de proposer des stratégies pour une meilleure conservation de cette importante ressource au Bénin.

Mots clés : Baobab, structure de population, distribution spatiale, conservation, Bénin. 


\title{
Characterization of some natural populations of Baobab (Adansonia digitata L.) and the pressures on them in the different chorological zones of Benin.
}

\begin{abstract}
Objective: Baobab is a multipurpose tree used by local populations in Benin. However, the conservation status of its populations remains little known. This study was undertaken to assess population's structure and to characterize the spatial distribution pattern of the species at Comè, Dassa and Matéri, localities belonging to the three chorological zones of Benin.

Methodology and results: Six hundred and sixty six (666) trees were studied in the three localities. A survey was conducted with 300 respondents in the local communities in the different localities to assess the threats due to anthropogenic pressures this plant is facing. Results revealed that Baobab populations are dominated by large trees (height $(15-20 \mathrm{~m})$, DBH 100-150 cm) with broad crown $(5-10 \mathrm{~m})$, and showed a weak regeneration especially in the young fallows and farms. The spatial distribution was overall random in the study areas. The type and intensity of threats vary from one study area to another, however, extensive agriculture constitutes the major threat reported.

Conclusion and application: The long-term maintenance of studied baobab populations will be compromised if the current anthropogenic pressures remain. Efforts must thus be made to lessen threats affecting the species mainly at its regeneration level. The results of this preliminary work provides baseline information that is necessary for future monitoring and detailed studies on these populations to analyze their evolution in the current context of heavy anthropogenic pressures and to propose strategies for better conservation of this important resource in Benin.
\end{abstract}

Key words: Baobab, population structure, spatial distribution, conservation, Benin.

\section{INTRODUCTION}

Les produits forestiers non ligneux sont utilisés à plusieurs fins par les populations africaines. Ils constituent un moyen de subsistance pour les populations à travers leur utilisation dans l'alimentation, en médecine mais sont aussi une importante source de revenu (Sven, 2006). Ces ressources provenant des écosystèmes forestiers sont sujettes à diverses formes d'exploitation et aux aléas climatiques. La dégradation des forêts est importante en Afrique de l'Ouest, où le défrichement annuel est estimé à $4 \%$ (FAO, 2001). Cette situation ne favorise pas l'expansion de certaines espèces des milieux forestiers et savanicoles. Au nombre de celle-ci figure le Baobab d'Afrique Adansonia digitata, une espèce endogène très importante au vu de la place centrale qu'elle occupe dans certaines sociétés (Codjia et al., 2003). A. digitata est un grand arbre de la famille des Malvacées et de l'ordre des Malvales pouvant atteindre 25 mètres de haut (Gebauer et al., 2002). Son tronc peut mesurer jusqu'à 10 mètres de circonférence, il est brillant, lisse, de couleur gris clair à foncé ou rougeâtre avec toute une gamme de couleurs intermédiaires (Terrible, 1991; Arbonnier, 2000). II est rencontré dans la plupart des régions semi-arides et subhumides au Sud du Sahara ainsi que dans l'Ouest de Madagascar, mais aussi dans les forêts de l'Afrique centrale où il est rare, puis près des côtes et des îles du Kenya et de la Tanzanie (Savard, 2003). C'est une espèce longévive à croissance lente pouvant atteindre 1200 ans (Patrut et al., 2007). Au Bénin, A. digitata est présent dans les trois zones chorologiques que compte le pays (Assogbadjo et al., 2005). C'est une espèce à usages multiples utilisée à des fins alimentaires, médicinales, culturelles, cultuelles et économiques (Codjia et al., 2002 ; Assogbadjo et al., 2006, 2009). L'espèce contribue de façon significative à la subsistance en milieu rural et à l'économie des populations locales (Sanogo et Tamba, 2012). Les feuilles sont consommées comme légume (Sidibe et al., 1996). La fibre de l'écorce peut être utilisée pour fabriquer des 


\section{Dossa et al. J. Appl. Biosci. 2015 Caractérisation de quelques peuplements naturels de Baobab (Adansonia digitata L.) et des pressions subies dans les différentes zones chorologiques du Bénin}

cordes. La pulpe brute et les graines sont respectivement transformées en poudre et huile destinées à l'exportation (Sanogo et al., 2015). C'est pourquoi de nombreuses questions se posent sur l'impact des divers types d'exploitation de l'espèce sur son état de conservation dans ses aires de distribution au Bénin. Cette étude

\section{MATÉRIELS ET MÉTHODES}

Milieu d'étude: Cette étude a été menée au Bénin, pays Ouest Africain situé entre les parallèles $6^{\circ} 30^{\prime}$ et $12^{\circ} 30^{\prime}$ de latitude Nord et le méridien $1^{\circ}$ et $3^{\circ} 40^{\prime}$ de longitude Est (Figure 1). La collecte des données a été réalisée dans les communes de Comé (département du Mono), Dassa (département des collines) et Matéri (département de l'Atacora) situées respectivement dans les trois zones chorologiques que compte le pays à savoir : (i) La zone Guinéo-Congolaise située entre $6^{\circ} 25^{\prime} \mathrm{N}$ et $7^{\circ} 30^{\prime} \mathrm{N}$ et caractérisée par quatre saisons ( 2 saisons pluvieuses et 2 saisons sèches). La pluviométrie moyenne est de $1200 \mathrm{~mm}$ par an avec une température moyenne journalière qui varie de $25^{\circ}$ à $29^{\circ} \mathrm{C}$. On rencontre dans cette zone des sols ferralitiques, profonds et peu fertiles, des sols alluviaux et vertisols. (ii) La zone Soudano-Guinéenne est située entre $7^{\circ} 30^{\prime} \mathrm{N}$ et $9^{\circ} 45^{\prime} \mathrm{N}$; c'est la zone de transition préalable a été menée afin d'apporter des éléments de réponse quant à la caractérisation de quelques peuplements de baobab au Bénin à travers la distribution spatiale, la structure des peuplements et les pressions anthropiques auxquelles l'espèce est confrontée.

entre la zone soudanienne et la zone guinéocongolaise. Le régime pluviométrique dans la zone soudano-guinéenne est unimodal (Mai-Octobre) et la pluviométrie moyenne annuelle varie de $900 \mathrm{~mm}$ à $1110 \mathrm{~mm}$ répartie le plus souvent sur 113 jours en moyenne. Les températures varient entre $25^{\circ} \mathrm{C}$ et $29^{\circ} \mathrm{C}$ dans cette zone. On y rencontre des sols minéraux peu évolués et peu fertiles et les sols ferrugineux sur socle cristallin (Natta, 2003). (iii) La zone Soudanienne située entre $9^{\circ} 45 \mathrm{~N}$ et $12^{\circ} 25 \mathrm{~N}$ a un régime pluviométrique unimodal avec une saison pluvieuse de juin à septembre et une saison sèche de novembre à mai. La pluviométrie dans cette zone varie de 900 à $1100 \mathrm{~mm}$ par an répartie en moyenne sur 145 jours. La température moyenne mensuelle varie de $24^{\circ} \mathrm{C}$ à $31^{\circ} \mathrm{C}$ dans cette zone. On y trouve des sols hydromorphes, des cuirasses ferrallitiques et des lithosols.

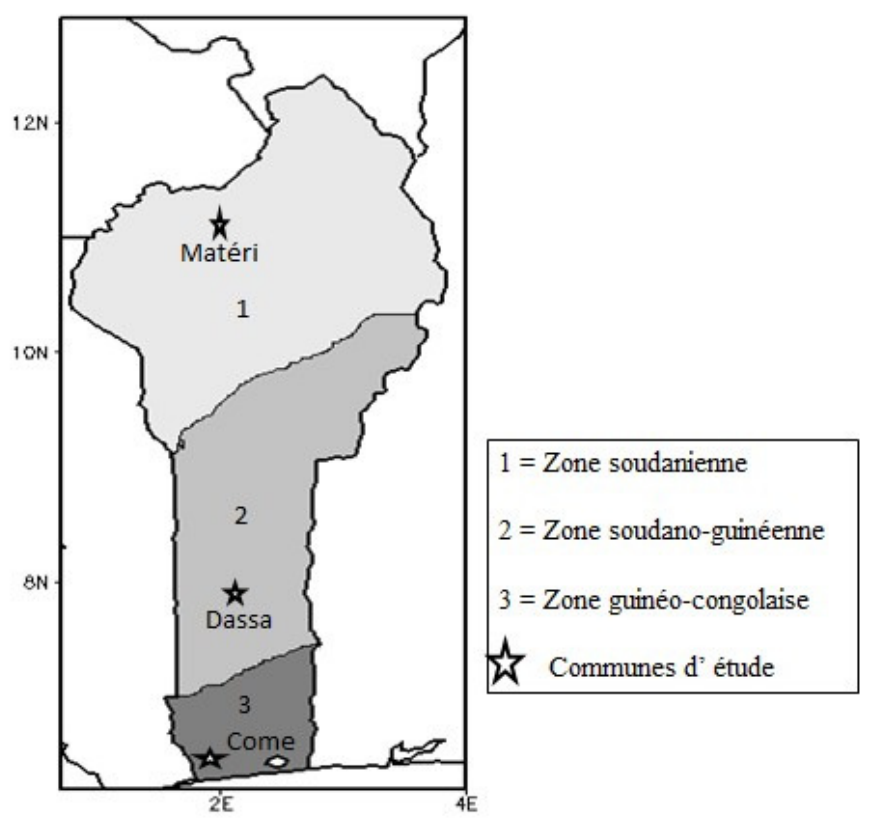

Figure 1. Carte présentant les zones chorologiques du Bénin et les communes d'étude 
Collecte des données : Des transects linéaires d'une longueur moyenne de $2 \mathrm{Km}$ ont été établis aléatoirement dans différents villages des communes d'étude où les pieds de baobab sont reconnus présents. Les pieds de baobab observés de part et d'autre des transects ont été recensés et étudiés. Dans la commune de Comé, les données ont été collectées dans 12 villages et 250 pieds de baobab ont été étudiés. Dans la commune de Dassa, 9 villages ont été parcourus et 251 sujets de baobab ont été étudiés. À Matéri, 165 sujets ont été étudiés dans 10 villages. Les données ont été collectées dans ces trois communes de Décembre 2011 à Février 2012. Ces données sont constituées des mesures dendrométriques (la hauteur, le diamètre à $1,30 \mathrm{~m}$ (DBH) et le rayon du houppier) des pieds de baobab rencontrés dans les communes d'étude et de leur géoréférencement (coordonnées UTM) à l'aide de GPS (Global Positioning System) de marque Garmin 60 de précision $5 \mathrm{~m}$. Une enquête a été menée auprès de 300 personnes vivant dans les environs des pieds de baobab à raison de 100 personnes par commune afin de recenser les formes de pressions qui pèsent sur l'espèce. Des entretiens semi-structurés ont été réalisés. Les critères d'échantillonnage sont: les groupes socioprofessionnels, les groupes socioculturels, les catégories d'âge et le sexe.

\section{Analyse des données}

Distribution spatiale : Le logiciel Programita (Wiegand et Moloney, 2004 version améliorée de 2007) a été utilisé pour l'analyse spatiale. La fonction de paircorrélation $G(r)$ (Stoyan et Stoyan, 1994) a été tournée. Ladite fonction utilise une forme normalisée de l'intensité pour apprécier les densités des points au voisinage de chaque point repère (ici correspond à un

\section{RÉSULTATS}

Caractérisation des pieds de baobab dans les peuplements: Le peuplement de baobab de la commune de Comè est représenté par des sujets ayant un diamètre (DBH) moyen de $159 \mathrm{~cm}$ (Figure 2). La régénération y est faiblement représentée. Les sujets sont majoritairement de grande taille $(15-20 \mathrm{~m})$ avec un recouvrement (rayon du houppier) de 6 à $8 \mathrm{~m}$. Les sujets ont été généralement rencontrés dans les champs et près des nombreuses carrières de graviers qui jonchent la zone d'étude et qui sont souvent des jachères. Dans la commune de Dassa, les pieds de baobab sont situés en majorité près des collines et dans les champs. Ce sont des arbres ayant une taille pied de baobab) et elle est fonction de la distance $r$ par rapport à ce point repère (Wiegand et Moloney, 2004).

La distance $r$ est une échelle qui est celle affichée sur l'axe des abscisses de la figure des résultats. Cette fonction est une version non cumulative de la fonction K de Ripley (Ripley, 1981) qui satisfait à l'équation :

\section{$G(r)=(1 / 2 \pi r) d K(r) / d r$}

(Avec $r$ l'échelle qui varie de 0 au maximum choisi qui est ici $50 \mathrm{~m}$ ). La dispersion spatiale finale ne dépend pas de la tendance de départ (à petite échelle) comme le fait la fonction $K$ de Ripley (Wiegand et Moloney, 2004) et cette fonction est plus intuitive que cumulative dans les mesures de densité (Stoyan et Penttinen, 2000) à cause de la normalisation. Pour l'interprétation des résultats, il y a une agrégation lorsque le résultat de l'analyse spatiale sort des intervalles de confiance au-dessus de la limite supérieure alors qu'il y a une régularité lorsque la sortie de la courbe de l'analyse spatiale est au-dessous de la limite inférieure. Enfin, il s'agit de distribution spatiale aléatoire lorsque le résultat de l'analyse spatiale reste à l'intérieur de l'intervalle de confiance.

Caractérisation des peuplements : Les données sur les paramètres dendrométriques collectés (le diamètre des arbres à hauteur de poitrine (DBH), la hauteur des arbres et le rayon du houppier des arbres) ont été rangées par classe et utilisées pour construire des histogrammes dans Excel 2007.

Données d'enquête: Les données de l'enquête ont été utilisées pour calculer les pourcentages des répondants pour chaque type de pression qui pèse sur les pieds de baobab sur les sites étudiés.

moyenne de 15, 4 m et un diamètre (DBH) moyen de $144 \mathrm{~cm}$. Le recouvrement (rayon du houppier) est en moyenne de 5,2 $\mathrm{m}$ (Figure 3). Une faible présence des individus de la régénération $(\mathrm{DBH} \leq 50 \mathrm{~cm})$ a été observée. Quant au peuplement de baobab de la commune de Matéri, il est composé majoritairement de pieds de diamètre (DBH) compris entre 150 et $250 \mathrm{~cm}$. Ces pieds ont été généralement rencontrés dans les espaces agricoles avec un recouvrement assez étendu (rayon du houppier : 6-10 m). Les sujets ont une taille moyenne de 14,5 $\mathrm{m}$ (Figure 4). La régénération y a été aussi faible mais mieux représentée comparée aux deux autres zones d'étude. 

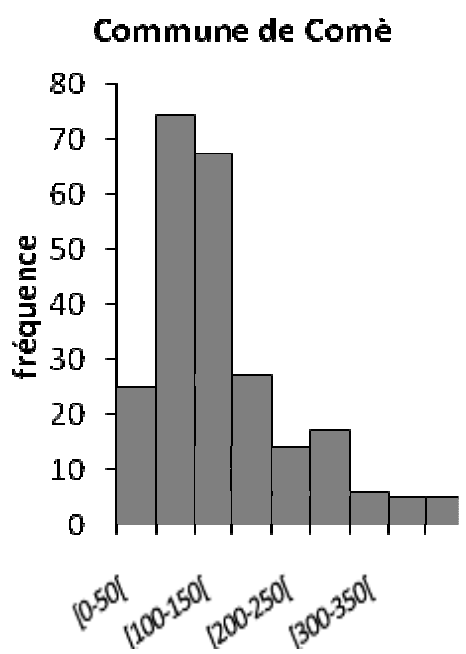

classes de diamètre $(\mathrm{cm})$
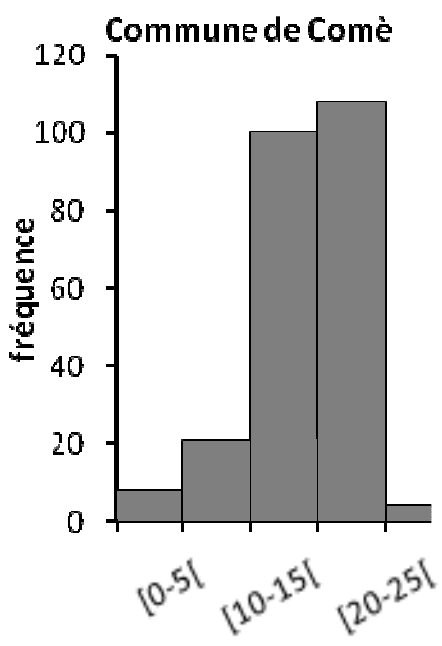

classes de hauteur (m)
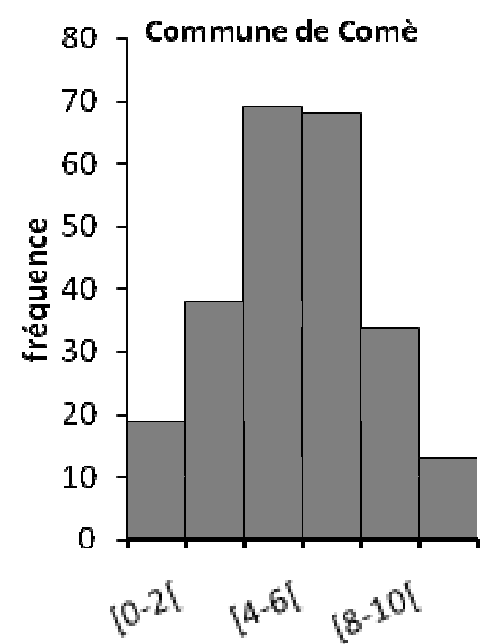

classes de ravon de houppier $\{\mathrm{m}\}$

Figure 2. Structure des classes de diamètre, de hauteur et de rayon de houppier des sujets de baobab de la commune de Comè
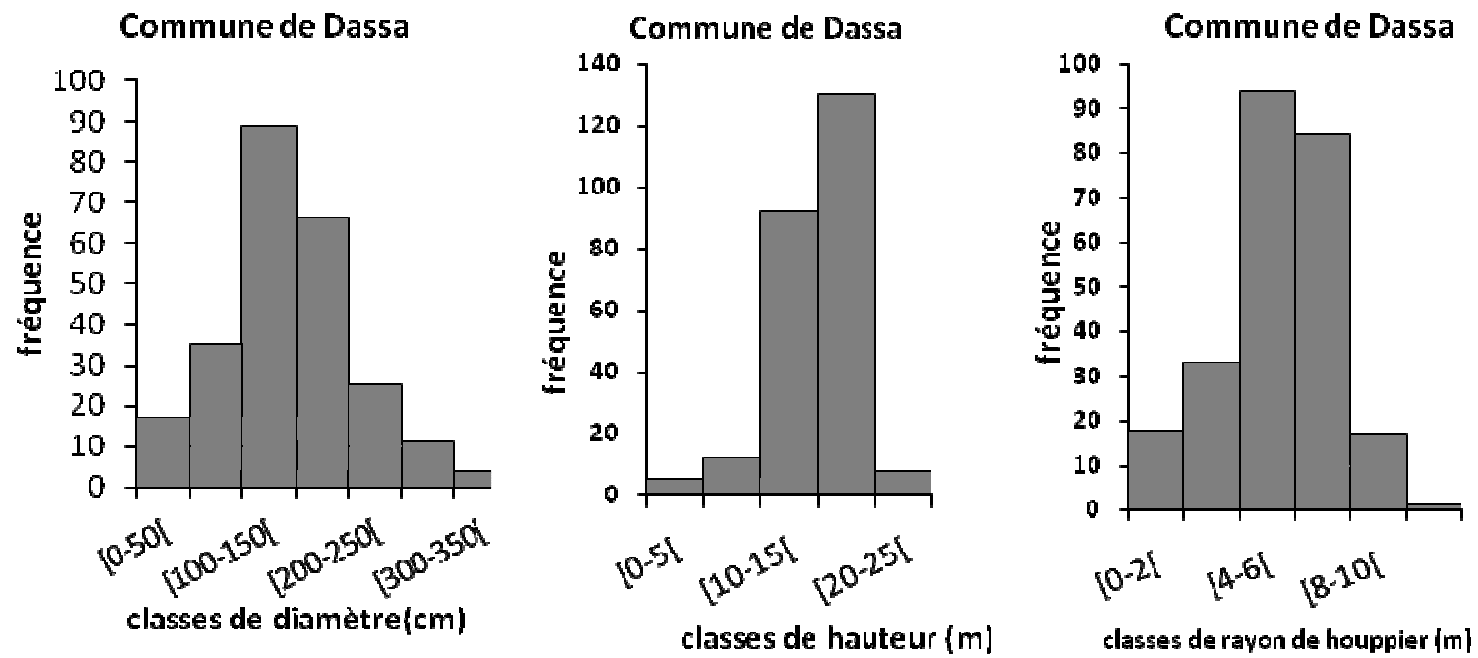

Figure 3 : Structure des classes de diamètre, de hauteur et de rayon de houppier des sujets de baobab de la commune de Dassa 


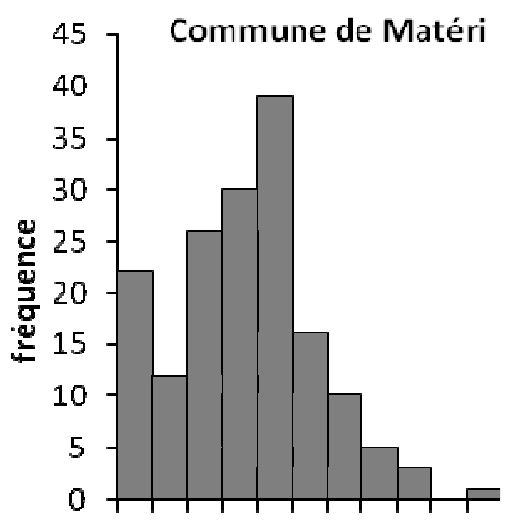

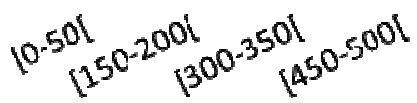

Classes de diamètre $\{\mathrm{cm}\}$
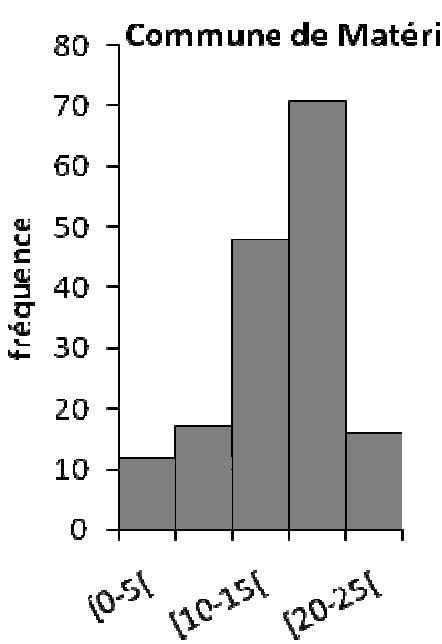

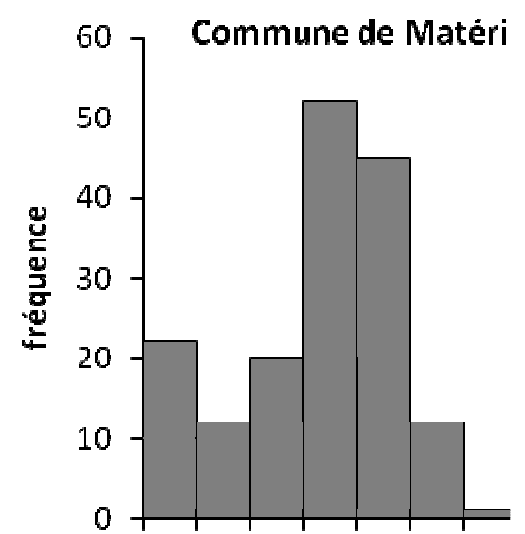

$0.2 l \quad 16.81 \quad 112.24 t$

Figure 4. Structure des classes de diamètre, de hauteur et de rayon de houppier des sujets de baobab de la commune de Matéri

Distribution spatiale: Les résultats de l'analyse spatiale sont présentés sur la Figure 5 . La courbe avec des cercles représente le résultat de l'analyse statistique des données, les courbes en trait plein représentent les limites supérieure et inférieure de l'intervalle de confiance. Les résultats de l'analyse statistique des données des différentes communes

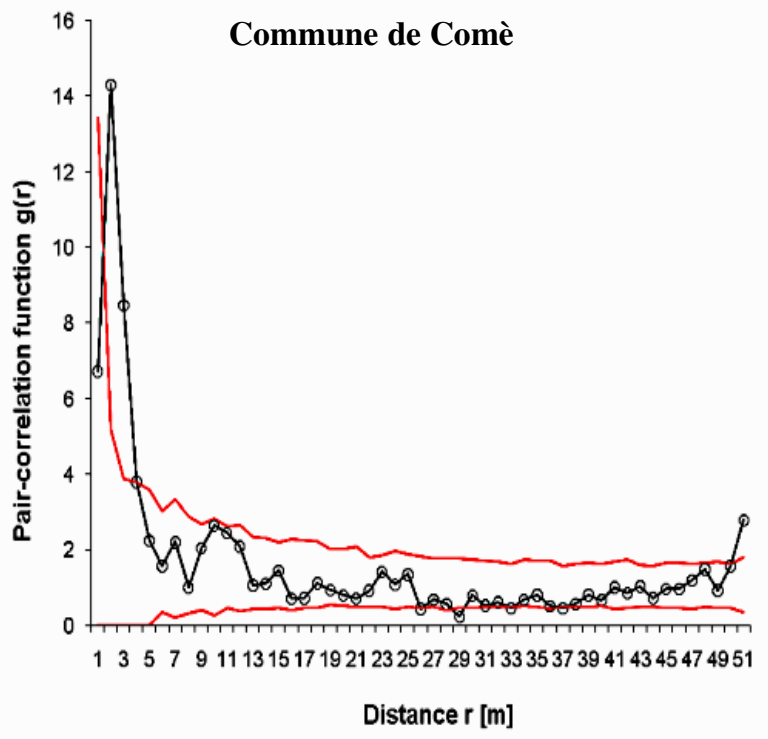

d'étude sont généralement situés à l'intérieur de l'intervalle de confiance. Ceci signifie que les pieds de baobab dans ces communes ont une distribution globalement aléatoire. On peut cependant remarquer une tendance à l'agrégation pour les faibles distances par rapport aux pieds repères $(r \leq 5 \mathrm{~m})$.

Commune de Matéri 


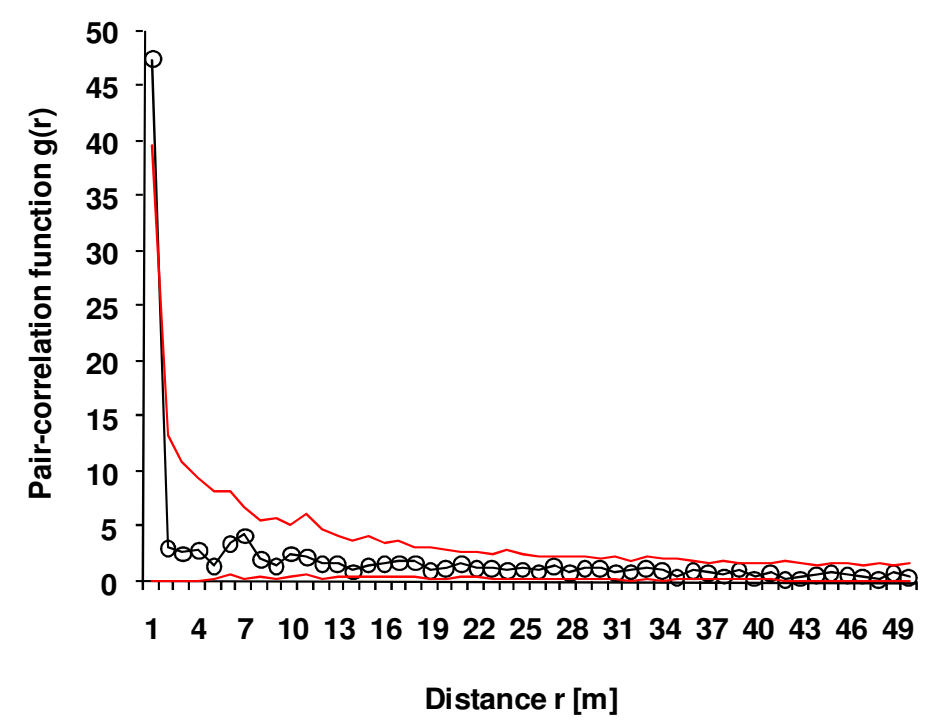

Figure 5. Distribution spatiale des baobabs dans les différentes communes d'étude

Différentes pressions recensées sur le baobab : Les pressions recensées lors de l'enquête ont été essentiellement d'ordres anthropiques (Tableau 1). Leur nature et leur intensité ont varié d'une région à une autre. La principale pression recensée sur l'espèce dans les trois communes d'étude a été l'agriculture extensive. Toutes les populations locales s'accordent sur le fait que le développement de l'agriculture avec la quête de nouvelles superficies cultivables affecterait considérablement l'effectif des peuplements de baobab.

Tableau 1. Pressions anthropiques recensées dans les différentes communes

\begin{tabular}{lccc}
\hline Pressions & $\begin{array}{c}\text { Commune de } \\
\text { Comè }\end{array}$ & $\begin{array}{c}\text { Communde de } \\
\text { Matéri }\end{array}$ & $\begin{array}{c}\text { Commune de } \\
\text { Dassa }\end{array}$ \\
\hline Agriculture & +++ & +++ & +++ \\
\hline Exploitation des capsules & - & + & - \\
\hline $\begin{array}{l}\text { Exploitation des carrières de } \\
\text { graviers }\end{array}$ & +++ & - & +++ \\
\hline $\begin{array}{l}\text { L'abattage dû au rôle supposé } \\
\text { dans les pratiques mystiques }\end{array}$ & +++ & + & + \\
\hline
\end{tabular}

\section{DISCUSSION}

La distribution spatiale des végétaux est une importante caractéristique des communautés écologiques (Jayaraman, 1999). Bien que cette analyse ne permet pas d'identifier directement les facteurs qui sont à la base de cette caractéristique des peuplements, elle peut permettre d'identifier les mécanismes sous-jacents et d'émettre des hypothèses (Legendre, 1993 ; Liebhold et al., 2002 ; Fajardo et al., 2006). L'absence de distribution agrégative ou régulière rapportée de l'analyse spatiale des peuplements de baobab dans les communes de Comè, Dassa et Matéri est conformes aux conclusions de Djossa et al. (2008) sur la distribution des pieds de Karité dans la région de la Pendjari au Nord-Ouest du Bénin. Cette distribution observée et surtout la faible densité des individus de la régénération pourraient être le fruit des activités anthropiques. En effet, la majorité des pieds de baobabs étudiés sont rencontrés dans des espaces agricoles. Kelly et al. (2004) ont conduit une étude similaire au Mali et ont rapporté que la distribution spatiale des sujets de karité, devenait de plus en plus agrégée des zones cultivées vers les forêts en passant 


\section{Dossa et al. J. Appl. Biosci. 2015 Caractérisation de quelques peuplements naturels de Baobab (Adansonia digitata L.) et des pressions subies dans les différentes zones chorologiques du Bénin}

par les vieilles jachères, preuve que les activités agricoles influencent le type de distribution spatiale de l'espèce. La faible tendance à l'agrégation observée à de faibles rayons parait normal vu l'envergure des rayons de houppiers (> $5 \mathrm{~m}$ ) rapportés sur les sujets de baobab. Cette tendance à l'agrégation à faible distance par rapport au pied repère, pourrait être supportée par la théorie de colonisation de Janzen (1970) et Connell (1971). Les peuplements de baobab des communes de Comè, Matéri et Dassa sont dominés par de grands sujets. Les différents graphes de distribution des diamètres révèlent une très faible proportion des sujets à faible diamètre. Ce qui traduit la rareté des jeunes sujets et plus particulièrement la régénération. Ces résultats confirment bien les conclusions de Assogbadjo et al. (2005). Soulignons que l'espèce n'est pas plantée au Bénin, ce qui pourrait être une explication supplémentaire à la proportion de régénération très faible observée. Dans les travaux de terrain de Chirwa et al. (2006) au Malawi et de Venter et Witkowski (2010) en Afrique du sud, aucune régénération de l'espèce n'a été observée. De plus, Venter et Witkowski (2013) ont montré que le baobab produisait suffisamment de graines viables, mais l'absence de conditions climatiques favorables à leur développent est le principal facteur à la faible régénération observée. Cette situation est préoccupante car, en l'absence d'une régénération naturelle suffisante, la survie de l'espèce sur le long terme est menacée. Les pressions subies par l'espèce sont surtout d'ordre anthropique et diffèrent selon les communes. Dans les trois communes, la principale source de pression exercée sur les peuplements de baobab est l'agriculture extensive. En effet, le baobab est perçu par les agriculteurs comme une espèce

\section{CONCLUSION}

De cette étude, il ressort que les peuplements de baobab des communes de Comè, Dassa et Matéri sont majoritairement constitués de sujets adultes avec une faible présence de la régénération. Cette situation pose un véritable problème car cette structuration peut perturber la dynamique des peuplements de l'espèce sur le long terme. Ils présentent également une distribution aléatoire avec une faible tendance à l'agrégation dans les trois communes. En dépit des conditions écologiques favorables à l'espèce, plusieurs formes de pressions notamment anthropiques compétitrice des cultures en raison de l'envergure de son houppier (> $10 \mathrm{~m}$ de diamètre) et de l'importance de l'espace qu'elle occupe dans les espaces cultivés. Ce point de vue des paysans n'est pas sans fondement car les travaux de Gbemavo et al. (2010) ont montré l'effet réducteur de l'ombrage du Karité sur la production capsulaire du coton. Les paysans n'hésitent donc pas à couper les pieds de baobab de leur champ et à éliminer très tôt les individus de la régénération. Les feux de végétation récurrents utilisés par les agriculteurs affectent également les jeunes plants de l'espèce. Ce résultat est conforté par les travaux de Venter et al. (2006) et la publication du Secrétariat de la Convention sur la Diversité Biologique (2002) qui ont montré que l'agriculture est une menace majeure pour la conservation de la biodiversité. Elle constitue la principale cause de la perte des habitats. En outre, plus de $60 \%$ des personnes enquêtées estiment que le baobab est un mauvais arbre car il est assez utilisé dans des pratiques mystiques. Cette considération socioculturelle ne milite pas en faveur de la conservation de l'espèce car elle est souvent abattue et éliminée aux alentours des agglomérations à l'état juvénile. L'espèce n'est pas non plus plantée par les populations pour les mêmes raisons. Savard (2003) a rapporté une attitude similaire des populations de Ségou au Mali qui ne sème pas l'espèce en raison de certaines croyances locales. Or depuis quelques années des travaux sont conduits dans la sous région Ouest Africaine (Burkina Faso, Mali, Niger, Bénin) en vue de la domestication de l'espèce (Jensen et al., 2011). La sensibilisation des populations concernées serait donc une étape cruciale dans le cadre de la vulgarisation de ces paquets technologiques.

menacent l'espèce et des efforts doivent être faits pour lever ces pressions afin de mieux valoriser l'espèce. Les résultats de ce travail posent les bases pour des études ultérieures de suivi régulier de ces peuplements afin d'analyser leur évolution dans le contexte actuel des pressions anthropiques encourues et de proposer des stratégies de conservation de cette importante ressource forestière au Bénin. Une extension de ces travaux vers d'autres peuplements de baobab au Bénin ainsi que l'impact de l'écorçage et de l'élagage sur ces peuplements méritent d'être menée. 


\section{Dossa et al. J. Appl. Biosci. 2015 Caractérisation de quelques peuplements naturels de Baobab}

(Adansonia digitata L.) et des pressions subies dans les différentes zones chorologiques du Bénin

\section{RÉFÉRENCES BIBLIOGRAPHIQUES}

Arbonnier M, 2000. Arbres, arbustes et lianes des zones sèches d'Afrique de l'ouest. Edition. CIRAD/MWHN/UICN France.

Assogbadjo AE, 2006. Importance socio-économique et étude de la variabilité écologique, morphologique, génétique et biochimique du baobab (Adansonia digitata L.) au Bénin. Thèse de doctorat. Faculty of Bioscience Engineering, Ghent University, Belgium. 213 p.

Assogbadjo AE, Sinsin B, Codjia JTC, Van Damme P, 2005. Ecological diversity and pulp, seed and kernel production of the baobab (Adansonia digitata) in Benin. Belgian Journal of Botany 138(1) : 49-56.

Assogbadjo AE, Sinsin B, De Caluwe E, Van Damme $P, 2009$. Développement et domestication du baobab au Bénin. LEA-FSA-UAC/DADOBAT, Cotonou, Bénin. 73 p. ISBN: 978-99919-6369-3.

Baum DA, 1995. The comparative pollination and fl oral biology of baobabs (AdansoniaBombacaceae). Annals of the Missouri Botanical Garden 82 : $322-348$.

Baum DA, Small RL, Wendel JF, 1998. Biogeography and floral evolution of baobabs (Adansonia, Bombacaceae) as inferred from multiple data sets. Syst. Biol. 47(2) : 181-207.

Chirwa M, Chithila V, Kayambazinthu D, Dohse C, 2006. Distribution and Population Structures of Adansonia digitata in Some Parts of Ntcheu, Dedza and Mangochi Districts, Malawi. FRIM, Zomba, Malawi.

Codjia JTC, Assogbadjo AE, Ekue MRM, 2003. Diversité et valorisation au niveau local des ressources forestières alimentaires végétales du Bénin. Cahiers Agricultures 12 : 321-331.

Codjia JTC, Fonton-kiki B, Assogbadjo AE, Ekue MRM, 2002. Le baobab (Adansonia digitata), une espèce à usage multiple au Bénin. CECODI /CBDD/ Veco/ SNV/ FSA. 47p.ISBN 99919953-0-7

Connell JH, 1971. "On the role of natural enemies in preventing competitive exclusion in some marine animals and in rain forest trees." In: Dynamics of Population. Ed. P.J. Den Boer and G.R. Gradwell. Wageningen: Pudoc. Wageningen, The Netherlands.

Djossa BA, Fahr J, Wiegand T, Ayihouénou BE, Kalko EKV, Sinsin BA, 2008. Land use impact on Vitellaria paradoxa C.F. Gaerten. Stand structure and distribution patterns: a comparison of Biosphere Reserve of Pendjari in Atacora district in Benin. Agroforestry System 72 : 205-220.

FAO, 2001. La situation des forêts et de la faune sauvage en Afrique. Commission régionale de la FAO pour l'Afrique. Rome, Italie.

FAO, 1993. Indigenous multipurpose trees of Tanzania: uses and economic benefits for people. Rome, Italie.

Fajardo A, Goodburn JM, Graham J, 2006. Spatial patterns of regeneration in managed unevenaged ponderosa pine/Douglas-fir forests of Western Montana, USA. For. Ecol. Manage. 223(1-3) : 255-266.

Gbemavo DSJC, Glèlè Kakaï $R$, Assogbadjo $A E$, Katary A, Gnanglè P, 2010. Effet de l'ombrage du karité sur le rendement capsulaire du coton dans les agroécosystèmes coton-karité du Nord Bénin. Tropicultura 28(4) : 193-199.

Gebauer J, El-siddig K, Ebert G, 2002. Baobab (Adansonia digitata): a Review on a Multipurpose Tree with Promising Future in the Sudan. Gartenbauwissenschaft 67 : 155-160.

Jayaraman K, 1999. Manuel de statistique pour la recherche forestière. FO: GCP/RAS/163/NET ; téléchargé http://www.fao.org/docrep/003/x6831f/x6831f1 8.htm

Janzen $\mathrm{DH}, 1970$. Herbivores and the Number of Tree Species in Tropical Forests. American Naturalist 104 : 940.

Jensen SJ, Bayala J, Sanou H, Korbo A, Ræbild A, Kambou S, Tougiani A, Bouda HN, Larsen A, Parkouda C, 2011. A research approach supporting domestication of Baobab (Adansonia digitata L.) in West Africa. New Forests 41 : 317-335 DOI 10.1007/s11056011-9246-z.

Kelly BA, Hardy JO, Bouvet J-M, 2004. Temporal and spatial genetic structure in Vitellaria paradoxa (shea tree) in an Agroforestry system in southern Mali. Molecular Ecology 13 : 12311240.

Legendre $P, 1993$. Spatial autocorrelation trouble or new paradigm? Ecology 74(6): 1659-1673.

Lewis SE, 1995. Roost fidelity of bats: a review. Journal of Mammalogy 76 : 481-496. 


\section{Dossa et al. J. Appl. Biosci. 2015 Caractérisation de quelques peuplements naturels de Baobab}

(Adansonia digitata L.) et des pressions subies dans les différentes zones chorologiques du Bénin

Liebhold AM and Gurevitch J, 2002. Integrating the statistical analysis of spatial data ecology. Ecography 25(5) : 553-557.

Maranz S and Wiesman Z, 2003. Evidence for indigenous selection and distribution of the shea tree, Vitellaria paradoxa, and its potential significance to prevailing parkland savanna tree patterns in sub-Saharian Africa, north of the equator. Journal Biogeography $30: 1505$ 1516.

Natta AK, 2003. Ecological assessment of riparian forests in Benin: phytodiversity, phytosociology and spatial distribution of tree species. PhD Thesis, Wageningen University. Netherlands, $215 \mathrm{p}$.

Patrut A, Von Reden K F, Lowy DA, Alberts AH, Pohlman JW, Wittmann R, Gerlach D, Xu L, Mitchell CS, 2007. Radiocarbon dating of a very large African baobab. Tree Physiology 27 : 1569-1574.

Ripley BD, 1981. Spatial statistics. New York, USA: Wiley.

Sanogo D et Tamba A, 2012. Inventaire des Parcs de Baobab et du potentiel pain de singe dans les CR de Koussanar et Bala dans la Région de Tambacounda et la CR de Dar Salam dans la Région de Kédougou. Rapport final d'étude MEA-USAl, Programme Agriculture Gestion des Ressources Naturelles «Wula Nafa» Sénégal 52p.

Sanogo D, Badji M, Diop M, Samb CO, Tamba A, Gassama YK, 2015. Évaluation de la production en fruits de peuplements naturels de Baobab (Adansonia digitata L.) dans deux zones climatiques au Sénégal. J. Appl. Biosci. $85: 7838-7847$.

Sidibé M, Williams JT, 2002. Baobab (Adansonia digitata). International center for underutilised crops, Southampton, UK.
Savard V, 2003. Evaluation du potentiel d'adoption des parcelles maraîchères de baobab (Adansonia digitata) dans la région de Ségou, au Mali. Mémoire de grade de Maître es Sciences (MSc), Université de Laval, Canada 128 p.

Secretariat of the Convention on Biological Diversity (SCBD), 2002. Review of the status and trends of, and major threats to, the forest biological diversity (CBD Technical Series no. 7). Montreal, SCBD, 164p.

Stoyan D and Stoyan H, 1994. Fractals, Random shapes and point fields. Methods of geometrical statistics. Wiley, Chichester.

Sven W, 2006. Le secteur PFNL en Afrique Centrale et l'importance du cadre légal et réglementaire. Limbé, Cameroun, 28 juin - 1 juillet 2006, présentation orale.

Terrible PB, 1991. Quelques arbres à multiplier dans les régions à longue saison sèche (savanes et Sahel). CESAO, Bobo-Dioulasso. $67 \mathrm{p}$.

Venter O, Brodeur N, Nemiroff L, Belland B, Dolinsek I, Grant J, 2006. Threats to endangered species in Canada. BioScience $56: 1-8$.

Venter SM et Witkowski TF 2010. Baobab (Adansonia digitata L.) density, size-class distribution and population trends between four land-use types in northern Venda, South Africa. Forest Ecology and Management 259 : 294-300.

Venter SM et Witkowski TF 2013. Using a deterministic population model to evaluate population stability and the effects of fruit harvesting and livestock on baobab (Adansonia digitata L.) populations in five land-use types. Forest Ecology and Management 303 : 113-120.

Wiegand $\mathrm{T}$ and Moloney K, 2004. Rings, circles and null-models for point pattern analysis in ecology. Oikos 104 : 209-229. 